\title{
PAPER
}

\section{Transient ischaemic attacks are associated with increased rates of global cerebral atrophy}

\section{R J L Walters, N C Fox, J M Schott, W R Crum, J M Stevens, M N Rossor, D J Thomas}

See end of article for authors' affiliations

Correspondence to: Dr D J Thomas, Department of Clinical Neurology, St Mary's Hospital, London W2 INY, UK; dafydd.thomas@ic.ac.uk

Received 30 July 2002 Accepted 22 October 2002

\begin{abstract}
Objectives: To determine whether patients presenting with a first transient ischaemic attack (TIA) subsequently show increased rates of brain atrophy compared with age matched controls; and to assess potential risk factors for brain atrophy in this group.

Methods: 60 patients with a first, isolated TIA and 26 age and sex matched controls were recruited. None had evidence of cognitive impairment. Vascular risk factors were treated appropriately. All subjects had volumetric imaging at the start of the study and one year later, when they were clinically reassessed. TIA patients also had serial dual echo brain imaging. Rates of whole brain atrophy were calculated from the registered volumetric scans, as was the incidence of new ischaemic lesions. In the TIA group, the degree of white matter disease was assessed. Atrophy rates and blood pressure were compared between patients and controls.

Results: 22 patients (37\%) developed new "clinically silent" infarcts during follow up. The mean (SD) annualised percentage atrophy rate in the TIA group was significantly higher than in the controls, at $0.82(0.39) \% \vee 0.33(0.3) \%(p<0.0001)$. In the TIA group, diastolic blood pressure $(p=0.004)$ and white matter disease severity $(p<0.001)$ were correlated with cerebral atrophy rate. Increased white matter disease was found in patients in whom new ischaemic lesions developed $(p<0.001)$.

Conclusions: Patients presenting with a first TIA have excess global brain atrophy compared with age matched controls over the subsequent year. Increased atrophy rates following a TIA may be directly or indirectly related to increasing white matter disease and diastolic hypertension. Future studies should assess whether this atrophy inevitably leads to cognitive decline, and whether aggressive treatment of risk factors for cerebrovascular disease (particularly hypertension) after a TIA can influence outcome.
\end{abstract}

C erebrovascular disease is associated with both cognitive decline and dementia. Cerebrovascular dementia may result from infarction of clinically eloquent cortical sites subserving specific cognitive functions or small, strategic subcortical infarcts (in the thalamus, caudate nucleus, or anterior internal capsule) that disrupt cortico-subcortical pathways. ${ }^{1}$ However, many patients with cerebrovascular disease present with a gradually progressive decline in cognition without the classical picture of stepwise decline resulting from discrete infarcts. While in these cases the pathological process leading to changes in cognitive function is not clear, it is likely that microscopic small vessel abnormalities lead to neuronal cell death. Magnetic resonance imaging in patients with vascular dementia may reveal widespread white matter signal change (leukoariosis) and increased rates of global cerebral atrophy, ${ }^{2}$ in common with other neurodegenerative disease such as Alzheimer's disease, where atrophy has been shown to correlate with cognitive decline and progression to dementia, ${ }^{3}$ and to be detectable before the onset of symptoms. ${ }^{45}$

Transient ischaemic attacks (TIA) are unequivocal evidence of cerebrovascular disease and constitute an increased risk of further cerebrovascular events or incipient coronary heart disease. ${ }^{67}$ A TIA might therefore also herald the onset of a process leading to cognitive decline at a time when the patient is still cognitively intact. Treatment of risk factors after a TIA substantially reduces the risk of subsequent stroke; a TIA might thus provide an important therapeutic window when intervention to prevent the development of cognitive impairment could be maximally beneficial. Furthermore, TIA are likely to represent the tip of the clinical iceberg, as small cerebrovascular lesions may not produce overt symptomatology but could contribute to the microvascular pathology that leads to cognitive decline.
In this study we assessed whether patients presenting with a first isolated TIA, despite treatment of vascular risk factors, had evidence of increased rates of cerebral atrophy in the absence of symptomatic stroke compared with age matched controls. We further assessed the risk factors and correlates of progressive brain atrophy in this group.

\section{METHODS \\ Subjects}

Sixty patients presenting to a neurovascular clinic with a first, isolated TIA were recruited to the study. All patients had suffered a TIA within the preceding 15 days. All were fully assessed by a consultant neurologist with a specialist interest in cerebrovascular disease. None had memory or other cognitive complaints and all had a Folstein mini-mental state examination (MMSE) ${ }^{9}$ score of at least 28/30. Current drug treatment and a smoking history were recorded. Seated blood pressure was measured from each arm to the nearest $2 \mathrm{~mm}$ $\mathrm{Hg}$; the higher reading was used for analysis. All patients had routine analyses of vitamin B-12, thyroid function, syphilis serology, glucose, and fasting cholesterol. An ECG and chest $x$ ray were done if clinically indicated. Patients with anterior circulation symptoms or features suggestive of carotid artery stenosis had duplex studies of the carotid vessels. All patients had serial volumetric and dual echo brain magnetic resonance (MR) imaging on the same scanner (see below). All patients were treated with antiplatelet agents (usually aspirin $75 \mathrm{mg}$ ), and also with cholesterol lowering agents (an HMG-CoA reductase inhibitor) if the fasting cholesterol was more than $6.5 \mathrm{mmol} / \mathrm{l}$. Antihypertensive drug treatment was continued.

The TIA patients were age and sex matched to 26 controls. None of the controls had subjective or objective evidence of cognitive impairment, and none had a history of stroke or 
symptoms suggestive of a TIA. All controls had volumetric brain MR imaging on one of two GE scanners (General Electric Co, Milwaukee, Wisconsin, USA). Serial scanning was done on the same machine. The presence of minor cerebrovascular change on MR imaging, judged by an experienced neuroradiologist to be within normal limits for age, did not exclude controls from the study.

Subjects were excluded from the study if there was:

- Subjective or objective evidence of cognitive impairment at recruitment (MMSE < 28/30);

- Radiological evidence of general or focal atrophy on initial MR imaging, as judged by an experienced neuroradiologist;

- Clinical or radiological evidence of established stroke;

- Alcohol consumption > 3 units daily;

- Severe hypertension requiring more than one change in antihypertensive treatment during follow up, or persistently raised diastolic blood pressure ( $>115 \mathrm{~mm} \mathrm{Hg}$ );

- Significant ischaemic heart disease, peripheral vascular disease, or carotid stenosis such that it was likely that major surgery might be required over the period of follow up.

All subjects gave informed written consent, and the study was approved by the local ethics committee. Patients and controls were reviewed at six months and one year and questioned specifically for the occurrence of new cerebrovascular events (TIA or stroke), and for the development of any new cognitive symptoms. Blood pressure was remeasured by the same investigator using the same protocol as at the start of the study.

\section{MR image acquisition and analysis}

All subjects had imaging at baseline and one year later on a 1.5 Tesla Signa unit (General Electric Co). At each scanning session, all TIA patients had both dual echo and volumetric imaging; the controls had volumetric imaging at each time point. Dual echo imaging was done with the following acquisition parameters: TE/TR/NEX/echo train, 90/4500/2/16; field of view, $24 \times 18 \mathrm{~cm}$. Volumetric imaging was done using a spoiled gradient echo technique (acquisition parameters: TR/TE/TI/theta, 17/4.2/450/20; field of view $24 \times 18 \mathrm{~cm}$; $256 \times 192$ image matrix). After acquisition, images were transferred to a Sun Sparc 10 workstation (Sun Microsystems) and analysed using the in-house MIDAS image analysis tool. $^{10}$

\section{Volumetric analysis}

For each subject, the follow up volumetric scan (at one year) was accurately registered onto the baseline image, using a nine degrees of freedom rigid body registration. Atrophy rates were calculated from the registered scan pairs using a previously validated tool, the brain boundary shift integral (BBSI). ${ }^{11}$

\section{Ischaemic changes}

For each individual, the difference image obtained from the registered scan pairs was studied in each of the axial, coronal, and sagittal planes to assess the incidence of new ischaemic lesions over the period of follow up.

White matter disease was scored for each patient at both time points by a consultant neuroradiologist (blinded to clinical diagnosis, scan order, or registration results) from the T2 weighted axially oriented images. A modification of Schelten's method $^{12}$ was employed. Using a scoring system between 0 and 6 , where 0 corresponds to no white matter change and 6 to confluent areas of change, the following areas were assessed: frontal lobes, parietal lobes, temporal lobes, occipital lobes, caudate, putamen, pallidum, thalamus, internal capsule, and pons. Thus for each subject and at each time point, a white matter score of between 0 and 60 was possible.

\begin{tabular}{llllll} 
Table 1 & \multicolumn{4}{l}{ Subject demographics } \\
\hline & $\mathrm{n}$ & Male & Age (years) & $\begin{array}{l}\text { Interval between } \\
\text { scans (months) }\end{array}$ \\
\hline TIA & 60 & 33 & $71.7(6.7)$ & $12.1(1.0)$ \\
Controls & 26 & 16 & $71.5(7.2)$ & $12.3(1.0)$ \\
\hline
\end{tabular}

Values are $\mathrm{n}$ or mean $(\mathrm{SD})$

TIA, transient ischaemic attack.

\begin{tabular}{|c|c|c|c|}
\hline & $\begin{array}{l}\text { Systolic BP } \\
(\mathrm{mm} \mathrm{Hg})\end{array}$ & $\begin{array}{l}\text { Diastolic BP } \\
(\mathrm{mm} \mathrm{Hg})\end{array}$ & $\begin{array}{l}\text { Cerebral atrophy } \\
\text { rate (\%/year) }\end{array}$ \\
\hline TIA & 152 (18.2) & 86 (11) & $0.82 \%(0.4)^{*}$ \\
\hline Controls & 146 (14) & $83.3(6.4)$ & $0.33 \%(0.3)$ \\
\hline \multicolumn{4}{|c|}{$\begin{array}{l}\text { Values are mean (SD). } \\
{ }^{*} p<0.001 v \text { control. } \\
B P \text {, blood pressure; TIA transient ischaemic attack. }\end{array}$} \\
\hline
\end{tabular}

\section{Statistical analysis}

Student's $t$ tests were used to compare atrophy rates and blood pressure between patients and controls. A regression technique was used to demonstrate any relation between atrophy and blood pressure in the patient group. A stepwise regression (entry criterion, $\mathrm{p}<0.05$ ) was used to correlate increasing blood pressure and white matter disease severity with cerebral atrophy rate, and sex with new lesion incidence. White matter disease severity scores were transformed to a normal distribution, using the equation:

$$
\text { new score }=[\text { white matter score }+1]
$$

and $t$ tests were used to compare white matter disease severity with the development of new ischaemic lesions in the patient group.

\section{RESULTS}

\section{Subject demographics and clinical characteristics}

Demographics of the patient and control groups are shown in table 1 . There was no significant difference in age between the patients and controls. In the patient group, 21 (35\%) were either past (previous year) or present smokers, four had diabetes mellitus (all with stable, good glycaemic control), and 14 had raised cholesterol concentrations. The latter were started on appropriate treatment.

Mean blood pressure (averaged from readings from the start and end of the study) for the patients and controls is shown in table 2. Patients had non-significantly higher systolic and diastolic blood pressure than controls.

Thirty three of the patients had TIA involving the anterior circulation and nine involving the posterior circulation; seven had isolated amaurosis fugax. The remaining 11 patients had experienced unilateral symptoms sparing the face and speech, and not clearly attributable to a single vascular territory. During the period of follow up, 12 patients had potentially significant medical events. One continued to have TIA despite maximum antiplatelet treatment, and went on to have an uneventful carotid endarterectomy. Four patients had syncopal episodes, one had an isolated seizure, one developed angina pectoris, three had pneumonia (necessitating admission to hospital), and two had unrelated operations requiring general anaesthesia.

The MMSE declined in three patients (from 29 to 27 in two, and from 29 to 26 on one). No control showed a decline in MMSE. 


\section{New lesion occurrence}

During follow up, 22 patients (37\%) developed new, small, clinically silent infarcts: nine developed one new silent lesion, four developed two lesions, three developed three lesions, four developed four lesions, one developed seven lesions, and the remaining patient developed 12 new lesions. One patient suffered a TIA during follow up (with an anatomically appropriate new ischaemic lesion seen on MRI), but none suffered a stroke. No new lesions were seen in the control group.

\section{Atrophy rates}

The mean (SD) annualised percentage atrophy rate in the TIA group was $0.82(0.4) \%$, and in the controls, $0.33(0.3) \%$ (table 2 ). Rates of cerebral atrophy were significantly higher in the patients than in the controls $(\mathrm{p}<0.0001)$.

In the TIA group, a positive correlation was found between increased rates of cerebral atrophy and systolic blood pressure $(p=0.02)$, diastolic blood pressure $(p=0.002)$, and the severity of white matter disease $(p<0.001)$. There was no correlation with age. Stepwise regression (entry criterion, $\mathrm{p}<0.05)$ showed that increasing diastolic blood pressure $(p=0.004)$ and white matter disease severity $(p<0.001)$ were correlated with cerebral atrophy rate.

A stepwise logistic regression technique showed that male sex was correlated with new lesion incidence $(p=0.006)$. Increased white matter disease was found in patients in whom new ischaemic lesions developed $(\mathrm{p}<0.001)$.

\section{DISCUSSION}

We have shown that patients presenting with a first isolated TIA have more than twice the rate of global brain atrophy as age matched controls over the subsequent year. This increased atrophy rate may reflect ongoing tissue damage at a subclinical level and would imply that these individuals are at higher risk of progressive cognitive decline.

Critical to this conclusion is determining the range of brain atrophy rates in normal controls and in patients with established dementia. Our finding of atrophy rates of $0.33 \%$ per annum in normal controls is broadly in keeping with the findings of Resnick et $a l^{13}{ }^{13}$ who reported that longitudinal atrophy rates based on ventricular volume changes were less than $0.5 \%$ per annum in aged controls, and of $\mathrm{O}^{\prime}$ Brien et $a l_{,}^{14}$ who reported annualised whole brain atrophy rates of $0.5 \%$ in 20 subjects using the BBSI technique. It is therefore likely that the excess atrophy we found in our patients following a TIA is a true finding; indeed TIA has been shown to be an independent risk factor for increased cortical atrophy in previous longitudinal computed tomography studies. ${ }^{15}{ }^{16}$ Rates of atrophy in established dementia are considerably higher than these values (for example, in Alzheimer's disease, 2.0 (0.9)\% per annum; in vascular dementia, $\left.1.9(1.1) \%{ }^{14}\right)$. It is therefore possible that, as none of the patients in this study had significant cognitive impairment, the excess atrophy we report might represent an intermediate stage between normal aging and the development of dementia. It is likely that there is considerable heterogeneity in our cases, as in most manifestations of cerebrovascular disease.

We have shown that patients with TIA have nonsignificantly higher blood pressures than age matched controls, supporting numerous studies where increasing hypertension is a risk factor for the development of cerebrovascular disease..$^{17}$ In our cohort, almost $40 \%$ of patients developed clinically silent ischaemic brain lesions during the first year of follow up, despite treatment with antiplatelet agents and, where appropriate, antihypertensive drugs. This finding supports the concept of a TIA being merely the tip of the iceberg as an indicator of cerebrovascular disease. Likewise, male sex is known to be an independent vascular risk factor and so the finding of an increased new lesion incidence in our male patients is not unexpected, and indeed has been reported before. ${ }^{16}$
We have shown that increased atrophy rates are related to increasing white matter disease. This might suggest that such lesions could be directly pathogenic, or might be markers of microscopic changes that lead to cell death and hence to atrophy. Alternatively, increased white matter disease may merely reflect the effect of hypertension. A recent longitudinal study established a relation between longstanding hypertension and the presence of white matter lesions, and showed that such changes might be prevented by adequate antihypertensive treatment. ${ }^{19}$ The relation between white matter hyperintensities and cerebral atrophy is unclear, and several previous studies have suggested independence between measures of white matter lesions and ventricular enlargement. ${ }^{20-22}$ However, in a longitudinal computed tomography study on elderly controls, Akiyama at al reported that progressive leukoariosis correlated directly with cortical atrophy and a decline in cortical perfusion. ${ }^{15}$ The same study reported that risk factors for increased cerebral atrophy included TIA, hypertension, and smoking. It therefore remains unclear whether white matter hyperintensities per se are directly or indirectly related to cerebral atrophy; indeed it is also apparent that many patients with extensive white matter lesions may be cognitively unimpaired $^{1}$ or indeed normotensive. ${ }^{19}$

We have shown that increasing diastolic hypertension is related to increasing rates of cerebral atrophy following a TIA. Hypertension has been found to be related to cerebral atrophy in cross sectional studies, ${ }^{2123}$ and hypertension in midlife has a direct relation to brain atrophy at necropsy, and to the accumulation of neurofibrillary tangles and neuritic plaques (the hallmarks of Alzheimer's disease). ${ }^{24}$ Hypertension is now thought to be an independent risk factor for Alzheimer's disease. ${ }^{25}$

There are therefore various possible explanations as to why hypertension might be associated with increased rates of cerebral atrophy. First, it could lead directly to neuronal dysfunction and death. Second, it might be one part of a cascade resulting in both covert and overt cerebrovascular disease (with or without white matter lesions or the clinical correlates of stroke or TIA), resulting in increased cerebral atrophy. Third, as hypertension appears to be an independent risk factor for Alzheimer's disease, the increased atrophy we observed may suggest the development of the earliest stages of Alzheimer's disease, with or without concomitant vascular dementia. Indeed in a recent study significant hippocampal neuronal loss, as occurs in Alzheimer's disease, was also found in patients with vascular dementia. ${ }^{26}$

The fall in MMSE score in a small number of patients (5\%) in the TIA group may be an early marker of the development of cognitive impairment over a longer period of follow up, in some patients possibly leading to dementia. Patients with brain atrophy and cerebrovascular disease have been reported to show a steeper decline in frontal executive function than controls, ${ }^{22}$ and change in ventricular volume correlates with cognitive measures in patients with established vascular dementia. ${ }^{27}$ A recent cross sectional study has shown that cognitive impairment in non-demented subjects was related both to the presence of white matter lesions and to cerebral atrophy. ${ }^{28}$ Longer term studies are required to assess fully the clinical correlates of the increased atrophy we report here. Such studies should include both detailed serial neuropsychology and volumetric MRI studies, assessing regional (for example, hippocampal) as well as global atrophy, to establish whether this excess atrophy inevitably leads to cognitive impairment, and if so, what are the relative contributions of vascular and Alzheimer's pathology.

A TIA is recognised to be a warning of the development of overt cerebrovascular and cardiovascular disease. We have shown that a TIA may also be predictive of subclinical cerebrovascular disease and of increased rates of cerebral atrophy which might in turn lead to cognitive impairment and possibly to dementia. There is increasing evidence to support 
aggressive treatment of hypertension in patients with cerebrovascular disease, ${ }^{29}$ and treatment with antihypertensive agents in elderly cognitively normal patients with isolated systolic hypertension appears to reduce the subsequent incidence of dementia. ${ }^{30}$

\section{Conclusions}

Our study supports the view that aggressive treatment of risk factors for cerebrovascular disease (particularly hypertension) is warranted after a single TIA. Future studies should assess whether such aggressive blood pressure management can halt or reduce excess brain atrophy after TIA, and whether such intervention reduces the long term risk of developing cognitive impairment or dementia.

\section{ACKNOWLEDGEMENTS}

This work was supported by the Stroke Association. We are grateful to Professor S Shorvon and Professor J Duncan for the opportunity to use the facilities of the National Society for Epilepsy. Statistical analysis was undertaken by Dr Paul Bassett, Imperial College School of Medicine Statistics Department, Du Cane Road, London. NCF is a Medical Research Council senior clinical fellow.

\section{Authors' affiliations}

R J L Walters, J M Schott, M N Rossor, D J Thomas, Department of Clinical Neurology, St Mary's Hospital, London W2, UK

N C Fox, W R Crum, Dementia Research Group, Institute of Neurology, Queen Square, London WCI, UK

J M Stevens, Department of Neuroradiology, The National Hospital for Neurology and Neurosurgery, Queen Square, London WC1, UK

\section{Competing interests: none declared}

\section{REFERENCES}

1 Haring HP. Cognitive impairment after stroke. Curr Opin Neurol 2002;15:79-84.

2 Bigler ED, Lowry CM, Anderson CV, et al. Dementia, quantitative neuroimaging, and apolipoprotein $\mathrm{E}$ genotype. Am J Neuroradiol 2000;21:1857-68

3 Fox NC, Scahill RI, Crum WR, et al. Correlation between rates of brain atrophy and cognitive decline in AD. Neurology 1999:52:1687-9.

4 Fox NC, Warrington EK, Rossor MN. Serial magnetic resonance imaging of cerebral atrophy in preclinical Alzheimer's disease. Lancet 1999;353:2125

5 Scahill RI, Schott JM, Stevens JM, et al. Mapping the evolution of regional atrophy in Alzheimer's disease: unbiased analysis of fluid-registered serial MRI. Proc Natl Acad Sci USA 2002;99:4703-7.

6 Dennis M, Bamford J, Sandercock P, et al. Prognosis of transient ischemic attacks in the Oxfordshire Community Stroke Project. Stroke 1990;21:848-53.

7 Hankey GJ, Slattery JM, Warlow CP. The prognosis of hospital-referred transient ischaemic attacks. J Neurol Neurosurg Psychiatry 1991;54:793-802.

8 Leys D, Deplanque D, Mounier-Vehier C, et al. Stroke prevention: management of modifiable vascular risk factors. J Neuro 2002;249:507-17
9 Folstein M, Folstein S, McHughs P. The "mini-mental state": a practical method for grading the cognitive state of patients for the clinician. $J$ Psychiatr Res 1975;12:189-98.

10 Freeborough PA, Fox NC, Kitney RI. Interactive algorithms for the segmentation and quantitation of 3-D MRI brain scans. Comput Methods Programs Biomed 1997:53:15-25.

11 Freeborough PA, Fox NC. The boundary shift integral: an accurate and robust measure of cerebral volume changes from registered repeat MRI. IEEE Trans Med Imaging 1997; 16:623-9

12 Scheltens $\mathbf{P}$, Barkhof $F$, Leys $D$, et al. A semiquantitative rating scale for the assessment of signal hyperintensities on magnetic resonance imaging I Neurol Sci 1993:114:7-12.

13 Resnick SM, Goldszal AF, Davatzikos C, et al. One-year age changes in MRI brain volumes in older adults. Cerebral Cortex 2000;10:464-72.

14 O'Brien JT, Paling S, Barber R, et al. Progressive brain atrophy on serial $M R I$ in dementia with Lewy bodies, $A D$, and vascular dementia. Neurology 2001;56:1386-8.

15 Akiyama H, Meyer JS, Mortel KF, et al. Normal human aging: factors contributing to cerebral atrophy. J Neurol Sci 1997;152:39-49.

16 Meyer JS, Rauch G, Rauch RA, et al. Risk factors for cerebral hypoperfusion, mild cognitive impairment, and dementia. Neurobiol Aging 2000;21:161-9.

17 MacMahon S, Peto R, Cutler J, et al. Blood pressure, stroke, and coronary heart disease. Part 1, Prolonged differences in blood pressure: prospective observational studies corrected for the regression dilution bias. Lancet 1990;335:765-74

18 Goldstein LB, Adams R, Becker K, et al. Primary prevention of ischemic stroke: a statement for healthcare professionals from the Stroke Council of the American Heart Association. Stroke 2001 :32:280-99.

19 de Leeuw F-E, de Groot JC, Ouderk M, et al. Hypertension and cerebral white matter lesions in a prospective cohort study. Brain 2002; 125:765-72.

20 Christiansen P, Larsson HB, Thomsen C, et al. Age dependent white matter lesions and brain volume changes in healthy volunteers. Acta Radiol 1994:35:117-22.

21 Salerno JA, Murphy DG, Horwitz B, et al. Brain atrophy in hypertension. A volumetric magnetic resonance imaging study. Hypertension 1992;20:340-8

22 Swan GE, DeCarli C, Miller BL, et al. Biobehavioral characteristics of nondemented older adults with subclinical brain atrophy. Neurology 2000:54:2108-14.

23 Hatazawa J, Yamaguchi T, Ito $M$, et al. Association of hypertension with increased atrophy of brain matter in the elderly. J Am Geriatr Soc 1984:32:370-4.

24 Petrovitch H, White LR, Izmirilian G, et al. Midlife blood pressure and neuritic plaques, neurofibrillary tangles, and brain weight at death: the HAAS. Honolulu-Asia Aging Study. Neurobiol Aging 2000;21:57-62.

25 Kivipelto M, Helkala EL, Laakso MP, et al. Midlife vascular risk factor and Alzheimer's disease in later life: longitudinal, population based study. BM 2001:322:1447-51.

26 Kril JJ, Patel S, Harding AJ, et al. Patients with vascular dementia due to microvascular pathology have significant hippocampal neuronal loss. J Neurol Neurosurg Psychiatry 2002;72:747-51

27 Broderick JP, Gaskill M, Dhawan A, et al. Temporal changes in brain volume and cognition in a randomized treatment trial of vascular dementia. J Neuroimaging 2001:11:6-12.

28 Koga H, Yuzuriha T, Yao H, et al. Quantitative MRI findings and cognitive impairment among community dwelling elderly subjects. J Neurol Neurosurg Psychiatry 2002;72:737-41.

29 PROGRESS Collaborative Group. Randomized trial of a perindopril-based blood pressure-lowering regimen among 6105 individuals with previous stroke or transient ischaemic attack. Lancet 2001;358: 1033-41.

30 Forette F, Seux ML, Staessen JA, et al. Prevention of dementia in randomised double-blind placebo-controlled Systolic Hypertension in Europe (Syst-Eur) trial. Lancet 1998;352:1347-51 